\title{
A influência do gênero e da escolaridade no diagnóstico de demência
}

\author{
The influence of gender and educational \\ level on the diagnosis of dementia
}

\author{
Cristina Lúcia Maia COELHO \\ Claudio Lyra BASTOS ${ }^{2}$ \\ Fernando Portela CAMARA ${ }^{3}$ \\ Jesus LANDEIRA-FERNANDEZ ${ }^{4,5}$
}

\begin{abstract}
Resumo
Um problema no diagnóstico das demências é a ausência de informação prévia sobre o nível cognitivo dos pacientes, anterior às manifestações atuais. A análise das discrepâncias nos testes cognitivos torna-se assim uma referência para o diagnóstico. A relação entre gênero, nível de escolaridade, experiência profissional e resultados da avaliação neuropsicológica foi analisada a partir de prontuários clínicos de pacientes idosos. A amostra masculina de nível superior apresentou médias significativamente menores nas subescalas de execução em relação às verbais. Apesar do nível de escolaridade e da atividade intelectual ocultarem a perda de performance nos quadros demenciais, a análise das discrepâncias evidencia as quedas de rendimento. Na amostra feminina, com menor nível de escolaridade, embora os resultados mostrassem deficiência cognitiva, não ocorreram diferenças significativas entre os subtestes verbais e de execução. A análise da covariância indicou que o nível de escolaridade, e não o gênero, foi o fator responsável pela diferença.
\end{abstract}

Unitermos: Avaliação neuropsicológica. Demência. Escolaridade. Gênero.

\begin{abstract}
A major problem with the early diagnosis of dementia is the lack of prior information about the cognitive level of patients before the current expression of the symptoms. The analysis of discrepancies in cognitive tests has become a reference for driving the diagnosis. Based on neuropsychological assessments of dementia in elderly patients, the presentstudy analyzed the relationship between gender, level of education and professional experience. The WAIS-III, Rey auditory verballearning, Folstein and Zülliger tests were employed. Results indicated that male subjects with higher education presented lower scores in the performancesubtest when compared to the verbal subtest. Although educational status and intellectual activity had a tendency to mask the loss of performance in dementia disorder, the evaluation of the discrepancies indicated a reduction in efficiency. In the sample offemales, with lower levels of schooling, although the results indicated a tendency towards cognitive impairment, no differences between verbal and performance subtests were detected. A covariance analysis indicated that the level of education was the variable responsible for this difference, and not gender.
\end{abstract}

Uniterms: Neuropsychological assessment. Dementia. Education status. Gender.

$\forall v$

1 Universidade Federal Fluminense, Faculdade de Educação. R. Major Fróes, 67, São Francisco, 24365-030, Niterói, RJ, Brasil. Correspondência para/ Correspondence to: C.L.M. COELHO. E-mail:<crismaia84@hotmail.com>.

2 Universidade Federal Fluminense, Hospital Universitário Antonio Pedro. Niterói, RJ, Brasil.

3 Universidade Federal do Rio de Janeiro, Instituto de Microbiologia Professor Paulo de Goés, Setor de Epidemiologia. Rio de Janeiro, RJ, Brasil.

- Pontifícia Universidade Católica do Rio de Janeiro, Departamento de Psicologia. Rio de Janeiro, RJ, Brasil.

5 Universidade Estácio de Sá, Curso de Psicologia. Rio de Janeiro, RJ, Brasil. 
O aumento mundial da expectativa de vida está diretamente ligado à incidência de doenças neurodegenerativas e alterações cognitivas significativas, sendo um dos fatores que justificam a urgência e importância das intervenções neuropsicológicas (Foss, Vale \& Speciali, 2005). As demências vêm apresentando um crescimento exponencial na sua taxa de prevalência sendo uma das principais causas de incapacitação no idoso (Lanska \& Schoenberg, 1993; Nitrini, 2002). A avaliação neuropsicológica se impõe nos quadros de declínio da capacidade intelectual, considerando as dificuldades no diagnóstico diferencial entre as alterações psicopatológicas que atingem a personalidade do idoso e comprometem o seu desempenho (Bastos, 2010).

A Neuropsicologia procura clarificar a relação existente entre funcionamento cerebral e atividades psicológicas superiores (como inteligência, percepção, memória, linguagem, atenção, entre outras), considerando tanto as variáveis biológicas quanto as socioculturais e emocionais. Atualmente, pode-se situá-la numa área de interface entre as Neurociências - neste caso, ela também pode ser chamada de Neurociência Cognitiva - e as Ciências do Comportamento, incluindo Psicologia do Desenvolvimento, Psicolinguística etc. (Lezak, 1995). O enfoque central é o estudo da relação entre o sistema nervoso, o comportamento e a cognição, investigando-se as capacidades mentais mais complexas (Gil, 2002). Considerando-se que o exame clínico nem sempre discrimina com precisão os déficits cognitivos, os testes neuropsicológicos investigam os elementos que compõem as funções corticais superiores, como a vigilância, a compreensão oral e leitura, entre outras (Snyder \& Nussbaum, 1999).

A Neuropsicologia clínica é frequentemente definida como uma ciência aplicada que trata das relações entre o comportamento e a disfunção cerebral. A Neuropsicologia estuda as funções do cérebro como um todo, cujas áreas são interdependentes e interrelacionadas, funcionando comparativamente a uma orquestra, que depende da integração de seus componentes para realizar um concerto. A isso se denomina sistema funcional. Dessa maneira sabe-se que, a partir do conhecimento do desenvolvimento e funcionamento normal do cérebro, podem-se compreender as alterações cerebrais, como os casos de disfunções cognitivas e do comportamento, resultantes de lesões, doenças ou desenvolvimento anormal do cérebro. Assim, a avaliação neuropsicológica deve ser concebida como uma ajuda ao diagnóstico diferencial dos transtornos neurológicos e psiquiátricos que afetam o funcionamento mental, como um método para se verificar a sua gravidade, e como um dos meios de acompanhar a sua evolução.

As demências, mesmo em sua fase inicial, provocam uma deterioração da eficiência cognitiva observável nos instrumentos de avaliação, isto é, queda do desempenho do sujeito se comparado com os resultados de antes do início do distúrbio. Em um estudo da avaliação executiva entre idosos, especificamente no que se refere à relação entre as demências e variáveis como gênero e escolaridade, Banhato e Nascimento (2007) encontraram uma diferença significativa em testes de fluência verbal, com melhor desempenho masculino. No mesmo estudo, a escolaridade afetou a velocidade de processamento, mas não a atenção, o raciocínio e a organização percepto-visuomotora. Analisando estudos de prevalência de demência em várias continentes, no período de 1994 a 2000, Lopes e Botino (2002) observaram maior prevalência de demência no sexo feminino, em todas as regiões pesquisadas. Em alguns estudos analisados, esse predomínio chegou à razão de duas mulheres para um homem. Houve predomínio do sexo feminino em 75\% dos estudos avaliados. Estudos realizados por Mota, Banhato, Silva e Cupertino (2008) com idosos indicam que o teste de triagem cognitiva Mini-Mental mostra-se menos útil na avaliação da demência no seu estado inicial quando comparado ao teste de Trilhas B, que revelou ser mais sensível. Os autores acrescentaram que o Mini-Mental é um teste altamente influenciado pela escolaridade, o que pode levar à identificação de falsos negativos (indivíduos em processo demencial, mas que não são identificados).

\section{A avaliação dos distúrbios da memória}

Um dos critérios do DSM-IV (1995) para demência é a alteração de memória de curto prazo e de longo prazo.

Deve-se distinguir a memória de evocação daquela de reconhecimento. Quando as amnésias de 
evocação são puras, o sujeito é capaz de reconhecer as próprias lembranças como sendo deles e como passadas, quando perguntas de múltipla escolha e indícios Ihe são propostos. Assim, o nome do presidente da república pode não ser evocável, mas reconhecido. As amnésias de dissolução do estoque mnêmico acabam com essa capacidade de reconhecimento das lembranças, quer elas digam respeito à memória social, quer à memória autobiográfica.

Segundo Gil (2002), na doença de Alzheimer os desempenhos são igualmente deficitários na rememoração e no reconhecimento. Assim, é danificada a memória de fatos antigos (remote memory, para os autores anglo-saxões) que, habitualmente, obedece a um gradiente temporal, e as lembranças são mais atingidas quando menos afastadas no tempo.

No subteste de Evocação do Mini-Mental (M. Folstein, S. Folstein \& McHugh, 1975), a lembrança de nome de três objetos, cinco minutos depois que eles foram citados, envolve o que se chama de possibilidade de aprender novas informações. É o que se denomina memória de curto prazo, não a imediata, mas a memória de fatos recentes ou memória secundária. A alteração da memória secundária é a mais típica em função da dificuldade do sujeito em adquirir, estocar e recuperar as novas informações. As relações profissionais, sociais e familiares acabam sendo perturbadas pelo esquecimento das compilações mnêmicas que tecem a vida cotidiana: encontro com pessoas, consultas, compras nas lojas, comunicações telefônicas, pagamentos de contas, fechar portas ou torneiras, guardar as chaves ou roupas.

\section{A neuropsicologia das demências}

A semiologia das síndromes demenciais não é única, mas múltipla. Tem a ver com a própria heterogeneidade das apresentações clínicas numa mesma etiologia. Fala-se num processo demencial porque nem sempre os critérios de desadaptação à vida profissional, social e familiar são reconhecidos inteiramente. Os sintomas iniciais podem ser queixas mnêmicas expressas pelo sujeito ou relatadas por familiares; também pode ser um desinvestimento progressivo dos interesses habituais. Combinados ou não com queixas do tipo depressivo, podem ser atos incongruentes, em desacordo com a personalidade habitual do sujeito.
Quadros demenciais, como o mal de Alzheimer, vêm associados a distúrbios do julgamento e de raciocínio, distúrbios de abstração e de interpretação de provérbios. Os testes de similitude, de vocabulário e de informação exploram a memória semântica, cujos déficits podem estar ligados ou não a uma deterioração do estoque das informações semânticas, ou a habilidade de acesso a essas informações. A demência de Alzheimer tende a comprometer a fluência literal e não a fluência categorial. Assim, mesmo que o paciente não consiga identificar numa salada os elementos de nome "tomate" ou "ervilhas", pode eventualmente lembrar-se de que pertencem à categoria dos "legumes" (Dartigues, Commenges \& Letenneur, 1997).

Ainda que se considere o postulado de que o cérebro constitui a base biológica do funcionamento mental, não se pode deixar de admitir o papel das relações sociais e do processo histórico no qual o homem está inserido. Com base nessa questão, torna-se fundamental a adoção da teoria sócio-histórica de Vygotsky \& Luria na compreensão do idoso no contemporâneo (Vygotsky \& Luria, 1998). O papel da cultura, das representações sociais e da educação nas avaliações neuropsicológicas vem sendo estudado (Foss et al., 2005), principalmente a influência da baixa escolaridade na avaliação das demências. Segundo pesquisas de Herrera, Caramelli, Silveira e Nitrini (2002), o gênero feminino e a baixa escolaridade associam-se significativamente à demência. Vale, no entanto destacar que, alguns estudos epidemiológicos entre diferentes culturas referem que pessoas com baixa escolaridade tendem a ser facilmente classificadas como pessoas com demência (Marcopulos, Mclain \& Guiliano, 1997). Assim, este trabalho assume o pressuposto de que o homem é tanto ser da natureza, quanto ser da cultura e instituidor da civilização (Jobim \&Souza,1994).

\section{Demência e depressão: o diagnóstico diferencial}

Alguns sintomas são comuns à demência e à depressão. A restrição do campo de interesses, a redução da atividade, a lentidão psicomotora que pode tocar as raias do apragmatismo e até a agitação psicomotora pertencem à semiologia comportamental tanto da demência quanto da depressão. A constatação de que a depressão pode provocar um déficit cognitivo, especial- 
mente distúrbio mnêmicos, gerou o termo pseudodemência. É importante não confundir pseudodemência (uma falsa demência) com demência reversível, que é uma demência real, mas passível de cura ou recuperação (Bastos, 2010).

Cabe ressaltar também a presença de depressão reativa ou secundária, de natureza emocional, a qual pode chegar a 20\% dos idosos, afetando a cognição e a motivação para a memória. A depressão comumente produz um déficit mnêmico, especialmente após os 40 anos, e esse prejuízo de memória do depressivo pode ser confundido com um quadro inicial de demência. Essa confusão depressão-demência pode ser maior ainda, levando-se em conta o fato de a depressão frequentemente ter características atípicas nos idosos (Gil, 2002). Embora possa haver comprometimento da disfunção cognitiva pela depressão, isso não exclui a presença de demência subjacente. No caso de idosos deprimidos, uma das principais características do exame neuropsicológico é a discrepância entre os resultados e as queixas apresentadas.

As habilidades mais preservadas no idoso são aquelas resultantes da aprendizagem substancial, isto é, habilidades bem consolidadas e, portanto, mais resistentes à deterioração mnêmica. Assim, avaliar as habilidades fluidas é um dos primeiros passos para o diagnóstico de demência. Em razão disso, o exame neuropsicológico pode também ser normal em alguns casos de demência incipiente, principalmente em indivíduos com elevada escolaridade. Isso ocorre porque, embora tenha ocorrido declínio de funções cognitivas, o desempenho ainda permanece dentro da faixa de normalidade. Sabe-se que a correlação entre a variável anos de estudo e o QI Total é de 0,76 e que tem um aumento progressivo até a faixa etária de 30-39 anos e ligeiro declínio entre as faixas de 40-49 e 65-89 anos (Kaufman 1990; Kaufman \& Lichtenberger 1999; Wechsler, 1997). Snyder \& Nussbaum (1999) admitem que um dos componentes significativos da avaliação neuropsicológica das demências refere-se a uma abordagem que possa considerar a história do paciente, assim como as circunstâncias do momento e as consequências na sua vida, em função dos resultados dos testes.

A validade do exame neuropsicológico na avaliação das demências seria assegurada caso se conhecessem os resultados anteriores ao início dos distúrbios, mas dificilmente isso ocorre. Assim, pode-se lançar mão das análises das discrepâncias dos resultados dos pacientes como pista. Segundo Roger Gil (2002), os resultados anteriores do sujeito podem ser supostos, se comparados os escores do sujeito em testes "resistentes" e testes "sensíveis". Os primeiros exploram mais o componente dito "cristalizado", que remetem à experiência e aos conhecimentos didáticos, como os testes verbais de vocabulário e de informação do WAIS-III. Já os segundos exploram o componente dito "fluido", que envolve a adaptação às novas situações, como os subtestes de execução como código ou os cubos do WAIS. Os primeiros estão muito ligados ao nível cultural, enquanto os segundos são mais independentes do nível cultural. Dessa forma, a existência da deterioração cognitiva poderá ser inferida.

O presente trabalho pretende analisar a relação entre variáveis como gênero, nível de escolaridade e experiência profissional de sujeitos idosos com seus desempenhos nos testes neuropsicológicos das subescalas verbais e de execução do WAIS-III e em outros testes neuropsicológicos.

\section{Método}

\section{Participantes}

Considerou-se a análise de resultados de testes neuropsicológicos em prontuários de 20 pacientes, 10 do sexo masculino e 10 do sexo feminino, com mais de 60 anos de idade, realizados em uma clínica de Saúde Mental privada em Niterói, visando na ocasião ao diagnóstico de demência. Vale registrar que os sujeitos foram selecionados a partir do critério idade (acima de 60 anos) e avaliados até determinado momento (outubro de 2007). No grupo de homens, todos possuíam $3^{\circ}$ grau de escolaridade. Na amostra feminina, 8 pacientes tinham menos de 4 anos de escolaridade e 2 entre 8 e 12 anos de estudo. Visando preservar as identidades dos pacientes, apenas os médicos psiquiatras e psicólogos que aplicaram os teste e os neurologistas tiveram ciência dos resultados.

\section{Instrumentos}

Tendo em vista a avaliação neuropsicológica dos transtornos cognitivos e demências, foram utilizados 
prontuários contendo os seguintes instrumentos: WAIS-III (Wechsler, 2004), a Lista de REY (Rey,1964), o Mini-Mental (Folstein et al., 1975), o teste projetivo Zülliger (Zülliger \& Salomon, 1970) e uma entrevista visando ao diagnóstico diferencial.

OWAIS-III permite avaliar a capacidade cognitiva, sendo constituído por subtestes que contribuem para os Ql's Verbal, de Execução e Total. O diagnóstico é desenvolvido a partir da análise das discrepâncias entre os escores do QI verbal e de execução, e também com base nos subtestes. Os subtestes verbais utilizados foram: a) informação; b) vocabulário; c) compreensão; d) semelhança (analogias); e) capacidade de cálculos; f) dígitos/números. Subtestes não verbais (QI execução), indicadores das habilidades executivas, referem-se à inteligência manipulativa, revelando a capacidade de integrar estímulos perceptuais e respostas motoras pertinentes. Os subtestes de execução utilizados foram: a) arranjo de figuras; b) completamento de figuras; c) cubos; d) código; e) montagem de objetos.

A lista de Rey (Auditory Verbal Learning Test - Rey, 1964) avalia a capacidade de aprender novas informações, retenção e reconhecimento. Consiste em cinco apresentações visando à aprendizagem de uma lista de 15 palavras, seguida de solicitação para que o paciente as repita, para avaliação da retenção imediata. Após 15 minutos é solicitada a lista visando à memória de retenção tardia. Por fim, visando ao reconhecimento, apresenta-se uma relação de 30 palavras, incluindo as 15 da lista original. Assim, uma curva de aprendizagem - ascendente ou não - é produzida dentro de um padrão de normalidade ou não para faixa etária do paciente (Lezak, 1995).

O Mini-Mental (Folstein et al., 1975) avalia funções como orientação, evocação, linguagem, aprendizagem, memória, cálculo, controle mental, compreensão e capacidade visuomotora. As entrevistas constaram de questões sobre a história de vida do paciente, incluindo aspectos da escolaridade, família e profissão. Assim, buscou-se criar um espaço transferencial favorável à ressignificação de histórias vividas no passado recente e remoto, às identificações, propiciando a construção de novas versões para experiências traumáticas, recentes ou antigas, inclusive as novas formas da imagem de si.

O Z-teste (Zülliger \& Salomon, 1970) permitiu avaliar: a) as condições de funcionamento psíquico e indícios de desintegração da personalidade; b) o grau de elaboração das respostas; c) o tipo vivencial e o modo de apreender a realidade; $d$ ) as possibilidades subjetivas do paciente quanto ao pragmatismo, à capacidade crítica, ao julgamento, atenção e ao grau de produtividade; e) a capacidade de relacionamento interpessoal; f) os índices de angústia.

A pesquisa obteve a aprovação do Comitê de Ética em Pesquisa do Departamento de Psicologia da PUC do Rio de Janeiro (Protocolo 02/2008).

\section{Resultados}

A partir de um levantamento quantitativo, pôde-se constatar que o diagnóstico da demência, ainda que considerando outras fontes clínicas como a história de vida do paciente, evidencia-se através das discrepâncias observadas entre resultados nas subescalas verbal e de execução do WAIS-III, nos resultados baixos no Rey (Auditory Verbal Learning Test) e na dimensão evocação do Mini-Mental.

No tocante à experiência profissional, em 100\% das entrevistas com os sujeitos do sexo masculino, foram destacados os seus percursos desde a conclusão do curso superior à prática profissional, com muita dedicação, obtenção de reconhecimento e respeito social, assim como garantia para o sustento da família. Considerando que todos já estavam aposentados, havia em seus discursos temas como: vida sem sentido após a aposentadoria, os filhos não precisam mais de mim, não há mais futuro, a falta de minha memória. Já nas entrevistas com as senhoras idosas, em suas falas havia referências muito positivas às suas atividades domésticas e artesanais, bem como menção a cuidados com os netos sem tom de queixa.

Numa análise comparativa entre pacientes do sexo masculino e feminino, encontraram-se médias significativamente superiores nas dimensões verbais entre os homens relativamente à amostra de mulheres. Nas dimensões manipulativas (testes de execução) não foram encontradas diferenças significativas entre as amostras feminina e masculina. A comparação entre homens e mulheres em relação a cada uma das variáveis do WAIS-III foi realizada através do teste $t$ Student.

Como homens apresentaram um nível de escolaridade maior em relação às mulheres, uma Análise de 
Covariância (ANCOVA) foi também realizada. Nesta análise, a variável escolaridade foi utilizada como fator de covariação em relação ao gênero dos sujeitos. Esta análise adicional permitiu verificar se o nível de escolaridade de nossa amostra poderia explicar eventuais diferenças significativas em relação às 11 variáveis dependentes analisadas, considerando-se estatisticamente significante a diferença em que $p<0,05$. A Tabela 1 apresenta as médias e os respectivos desvios-padrão de homens e mulheres em relação às 11 variáveis avaliadas no WAIS-III.

Como se pode observar na Tabela 1, a amostra composta por homens apresentou médias sistematicamente superiores em todas as variáveis em relação à amostra formada por mulheres. Entretanto, o teste $t$ Student revelou que apenas as variáveis Informação $(t=3,41 ; p<0,01)$, Aritmética $(t=3,01 ; p<0,01)$, Vocabulário $(t=2,81 ; p<0,05)$ e Dígito-Memória $(t=2,71 ; p<0,01)$ apresentaram uma diferença significativa. Deve-se notar que as diferenças significativas encontradas nessas quatro variáveis não podem ser atribuídas ao gênero dos sujeitos.

Análises adicionais com a ANCOVA, em que a escolaridade foi utilizada como fator de covariação, indicou ausência de diferença significativa entre os grupos, não só nestas quatro variáveis, mas também nas sete restantes.

Esses resultados sugerem que as diferenças significativas entre homens e mulheres detectadas através do teste $t$ Student estão associadas ao grau de escolaridade da amostra pesquisada. Encontraram-se, na amostra de pacientes do sexo masculino de nível superior, médias significativamente mais baixas nas subescalas de execução do WAIS-III quando comparadas às médias das subescalas verbais. Ou seja, há uma tendência a haver maior discrepância entre os fatores verbais e execução, em detrimento dos últimos, entre homens de nível superior.

Na amostra feminina com pacientes de nível básico de escolaridade, não se encontrou diferença significativa entre os resultados verbais e não verbais. No caso da amostra feminina, não houve discrepâncias significativas no WAIS-III, sendo necessários outros testes, como a lista de Rey e o Mini-Mental, para identificar o processo demencial.

Relativamente ao estudo da memória, foram encontrados porcentagens significativamente altas entre os pacientes, tanto do sexo masculino quanto do feminino, com resultados baixos (abaixo de escore ponderado 8) no subteste verbal de dígitos (94\%), na dimensão evocação do Mini-Mental (66\% não acertaram o item evocação) e também abaixo da faixa de normalidade na memória de evocação e de estocagem na lista de Rey (90\%). Igualmente, os índices de memória de reconhecimento na lista de Rey tenderam a ser altos (amnésia por dissolução do estoque), tanto na amostra feminina como na masculina. Não foram encontradas alterações psicopatológicas da personalidade em nenhum dos casos, nem transtornos depressivos significativos, embora 30\% do grupo masculino apresentassem sinais de depressão e angústia.

Tabela 1. Médias e desvios-padrão dos resultados nos sub-testes (variáveis) nas amostras de homens e mulheres.

\begin{tabular}{|c|c|c|c|c|c|c|c|}
\hline & \multirow{2}{*}{ Variável } & \multicolumn{2}{|c|}{ Homens } & \multicolumn{2}{|c|}{ Mulher } & \multicolumn{2}{|c|}{ Teste de hipótese } \\
\hline & & Média & Desvio-padrão & Média & Desvio-padrão & Teste $t$ student & Análise de covariância \\
\hline & Informação & 12,8 & 2,9 & 7,9 & 2,7 & $p<0,01$ & $p<0,12$ \\
\hline$\cap$ & Semelhanças & 12,6 & 4,1 & 9,8 & 3,4 & $p<0,12$ & $p<0,82$ \\
\hline 3 & Aritmética & 11,3 & 3,9 & 7,0 & 2,2 & $p<0,01$ & $p<0,37$ \\
\hline \multirow{3}{*}{ 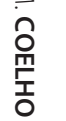 } & Vocabulário & 14,0 & 3,3 & 11,3 & 2,5 & $p<0,05$ & $p<0,28$ \\
\hline & Compreensão & 12,7 & 6,5 & 8,3 & 5,2 & $p<0,12$ & $p<0,33$ \\
\hline & Dígito-memória & 10,1 & 3,2 & 6,7 & 2,3 & $p<0,01$ & $p<0,91$ \\
\hline$\stackrel{1}{+}$ & Arranjo de figuras & 8,1 & 5,8 & 7,0 & 4,6 & $p<0,64$ & $p<0,32$ \\
\hline 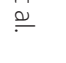 & Completar figuras & 9,8 & 5,2 & 8,3 & 3,7 & $p<0,64$ & $p<0,88$ \\
\hline 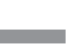 & Cubos & 9,1 & 6,3 & 5,7 & 4,6 & $p<0,18$ & $p<0,54$ \\
\hline & Armar objetos & 7,2 & 2,5 & 6,4 & 3,9 & $p<0,59$ & $p<0,58$ \\
\hline 4 & Código & 9,6 & 2,3 & 7,5 & 4,7 & $p<0,31$ & $p<0,12$ \\
\hline
\end{tabular}




\section{Considerações Finais}

\section{O envelhecimento como construção psicossocial}

Não obstante o número reduzido da amostra, que limita a sua validade externa e restringe a aplicação dos resultados, foi possível verificar que é o nível de escolaridade, e não o gênero, o fator que explica as diferenças de desempenho entre homens e mulheres idosos em testes neuropsicológicos. Esses resultados podem ser compreendidos a partir de fatores culturais e simbólicos, característicos de cada grupo: de um lado, a experiência profissional dos homens de nível superior, baseada em práticas que envolviam discursos, linguagem e comunicação; de outro, as práticas domésticas das mulheres sem curso superior - incluindo costura, culinária e outras atividades artesanais -, que envolviam aspectos tanto verbais como visuoespaciais. Esses dados corroboram estudos que encontram diferenças significativas entre diferentes grupos educacionais (Marcopulos et al., 1997; Rosselli, Ardila \& Rosas, 1990).

Relativamente aos aspectos motivacionais, encontraram-se traços depressivos em maior frequência entre a amostra masculina com demência, do que na amostra feminina. Pode-se interpretar esse fato a partir da desvalorização sentida pelos homens em relação aos papéis sociais e familiares que ocupavam anteriormente, assim desenvolvendo menor tolerância à dependência que os déficits cognitivos trouxeram para suas vidas.

A validade do exame neuropsicológico na avaliação das demências foi atingida, na medida em que a análise revelou uma discrepância significativa nos resultados dos pacientes da amostra masculina - todos de nível superior: de um lado, os escores dos testes "resistentes", que envolvem testes verbais de vocabulário e de informação do WAIS-III e estão ligados à experiência escolar e profissional; e, de outro, os resultados nos testes "sensiveis", que exploram o componente dito "fluido", mediante os subtestes de execução como código, cubos e dígitos, mais independentes do nível educacional.

Por fim, vale ressaltar que a simples perda de desempenho cognitivo não dá indicação etiológica, podendo resultar de um dano orgânico cerebral, de um estado depressivo ou de uma síndrome psicótica. Há um ponto que atravessa o trabalho com os idosos: a importância de ser consolidada uma nova concepção de envelhecimento, diferente daquela que enfatiza a dependência e a tendência inexorável à degenerescência, como a indicar que existiria uma ordem fixa para esse processo: degradação da inteligência, da afetividade, da memória e da capacidade de trabalhar. Podem-se considerar duas orientações dominantes quanto à questão (Néri, 2001):

a) aquela que define o idoso em um contexto semântico depreciativo e pessimista, enfatizando os efeitos degenerescentes da passagem do tempo e pregando ações que tenham tem como objetivo os cuidados com o corpo, com o recurso à fisioterapia e à farmacologia;

b) aquela que promove uma consideração histórica do desenvolvimento, colocando em destaque a adaptação do idoso às representações estabelecidas, vinculando seu conceito às formas de tratamento e cuidado que recebe.

No sócio-historicismo, Vygotsky e Luria (1998) situam diferentemente os "processos reflexos", que não supõem aprendizagem, dos "processos mentais superiores", que dependem das relações sociais, por sua vez norteadas por representações sociais. A memória, a linguagem, a capacidade para trabalhar e se reorganizar, não são processos naturais, mas essencialmente culturais, cabendo, portanto, uma variação bem significativa que cabe explorar e expandir. Essas variações podem ser aproximadas às práticas sociais concretas, aos procedimentos rotineiros que propiciam identificação ao idoso, promovendo seu estilo de amar, criar e trabalhar.

Assim entende-se que, mesmo havendo comprometimento orgânico em nível cerebral, tal fato não descarta o alcance da significação concedida a essa perda. Em síntese, não se trata apenas de cuidar da demência, mas da velhice, que merece ser entendida como uma construção social produzida pela sociedade atual, na qual a aura simbólica de experiência e sabedoria de que o idoso desfrutava nas sociedades tradicionais parece se esvair, pouco havendo a transmitir para as novas gerações.

\section{Referências}

Bastos, C.L. (2010). Manual do exame psíquico: uma introdução prática à psicopatologia (3a ed.). Rio de Janeiro: Revinter. 
Banhato, E., \& Nascimento, E. (2007). Função executiva em idosos: um estudo utilizando subtestes da Escala WAIS-III. PSicoUSF, 12 (1), 65-73.

Dartigues, J., Commenges, D., \& Letenneur, D. (1997). Cognitive predictors of dementia in elderly community residents. Neuroepidemiology, 16 (1), 29-39.

DSM-IV. (1995). Manual diagnósticoeestatístico de transtornos mentais (4 ed.). Porto Alegre: Artes Médicas.

Folstein, M., Folstein, S., \& McHugh, P. R. (1975). Mini-Mental State: a practical method for grading the cognitive state of patients. Journal of Psychiatric Research, 12, 189-198.

Foss, M.,Vale, F., \& Speciali, J. (2005). Influência da escolaridade na avaliação neuropsicológica de idosos. Arquivos de Neuropsiquiatria, 63 (1),119-126.

Gil, R. (2002). Neuropsicologia. São Paulo: Santos editora.

Herrera, J., Caramelli, P., Silveira A., \& Nitrini, R. (2002). Epidemiologic survey of dementia in a communitydwelling Brazilian population. Alzheimer Disease and Associated Disorders, 16 (2), 103-108.

Jobim \& Souza, S. (1994). Infância elinguagem: Baktin, Vygotsky e Benjamim. São Paulo: Papirus.

Kaufman, A. (1990). Assessing adolescent and adultintelligence. Boston: Allyn \& Bacon.

Kaufman, A., \& Lichtenberger, E. (1999). Essentials of WAIS-III Assessment. New York: John Wiley \& Sons.

Lanska, D.J., \& Schoenberg, B.S. (1993). The epidemiology of dementia: methodologic, issues and approaches. In Whitehouse PJ. Dementia (pp.3-22). Philadelphia: FA Davis Company.

Lezak, M. (1995). Neuropsychological assessment (2 ${ }^{\text {nd }}$ ed.). Oxford: Oxford University Press.

Lopes, M., \& Botino, C. (2002). Prevalência de demência em diversas regiões do mundo: análise dos estudos epidemiológicos de 1994 a 2000. Arquivos de Neuro-Psiquiatria, 60 (1), 61-69.
Marcopulos, B., Mclain, C., \& Guiliano, A. (1997). Cognitive impairement or inadequate norms? A study of health, rural, older adults with limited education. Clinical Neuropsychological, 11 (2), 111-131.

Mota, M., Banhato, E., Silva, K., \& Cupertino, A. (2008). Triagem cognitiva: comparações entre o mini-mental e o teste de trilhas. Estudos em Psicologia (Campinas), 25 (3), 353-359.

Néri, A. (Org.) (2001). Maturidade evelhice: trajetórias individuais e socioculturais. Campinas: Papirus.

Nitrini, R. (2002). Demência e doença de Alzheimer no Brasil. Jornal Brasileiro de Neuropsiquiatria Geriátrica, 3 (1), 6-7.

Rey, A. (1964). L'exame clinique en psychologie. Paris: Press Universitaires de France.

Rosselli, M., Ardila, A., \& Rosas, P. (1990). Neuropsychological assessment illiterates: II language and praxis abilities. Brain and Cognition, 12, 281-289.

Snyder,P.J., \&Nussbaum, P.D. (1999). Clinical neuropsychology: a pocket handbookfor assessment. Washington: American Psychology Association.

Vygotsky, L., \& Luria, A. (1998). Linguagem, desenvolvimento e aprendizagem. São Paulo: Ícone.

Wechsler, D. (1997). Wechsler adult intelligence scale (3 ${ }^{\text {rd }}$ ed.). San Antonio, TX: Psychological Corporation.

Wechsler, D. (2004). WAIS-III Escala de inteligência Wechsler para adultos: manual David Wechsler. Adaptação e padronização de uma amostra brasileira. São Paulo: Casa do Psicólogo.

Zulliger, H., \& Salomon, F. (1970). El Test de Zulliger. Buenos Aires: Kapelusz.

Recebido em: 29/4/2008

Versão final reapresentada em: 27/8/2010

Aprovado em: 9/9/2010 